\title{
Comprehensive circular RNA profiling reveals the regulatory role of circ_100242/miR-145 pathway in bladder cancer
}

\author{
ZHAOWEI ZHU, FUJIANG CHANG, JUNXIAO LIU, JIANGE WANG and XUEPEI ZHANG \\ Department of Urology, The First Affiliated Hospital of Zhengzhou University, Zhengzhou, Henan 450052, P.R. China
}

Received January 6, 2019; Accepted September 11, 2019

DOI: $10.3892 / \mathrm{ol} .2020 .11380$

\begin{abstract}
Bladder cancer is a common genitourinary carcinoma with high morbidity and mortality rates. Circular RNAs (circRNAs), which are a type of single-stranded RNAs, have been characterized as stable, conserved and tissue-specific molecules in mammalian cells. The present study explored the circRNA expression profile in four bladder cancer tissues and matched normal samples by using microarray analysis. Furthermore, bioinformatics analyses were performed to investigate the potential function of dysregulated circRNAs in bladder cancer. The results demonstrated that 89 circRNAs were downregulated and 210 circRNAs were upregulated in bladder cancer tissues. The results from RT-qPCR demonstrated that hsa_circ_100241,hsa_circ_100242 and hsa_circ_101303 were markedly upregulated whereas hsa_circ_104510 was significantly downregulated in bladder cancer tissues compared with normal tissue. Following circRNA/microRNA (miRNA) interaction network generation via Cytoscape, it was demonstrated that hsa_circ_100242 contained a miRNA response element for miR-145-5p, which is a tumor suppressor in bladder carcinoma. In addition, results from the Kyoto Encyclopedia of Genes and Genomes analysis showed that the MAPK signaling pathway was the most significant pathway of the differentially expressed circRNAs in bladder cancer tissues. In conclusion, the present study demonstrated that circRNAs were dysregulated in bladder cancer tissues compared with matched normal samples. Pathway analysis was also performed to predict the binding of miRNAs to the dysregulated circRNAs. The results revealed that hsa_circ_100242 may be involved in bladder cancer initiation and progression by sponging miR-145. These findings may provide further insights into the functional and therapeutic roles of circRNAs in bladder cancer.
\end{abstract}

Correspondence to: Dr Zhaowei Zhu or Professor Xuepei Zhang, Department of Urology, The First Affiliated Hospital of Zhengzhou University, 1 East Jianshe Road, Erqi, Zhengzhou, Henan 450052, P.R. China

E-mail: zzwdoctor6@126.com

E-mail: zhangxuepei@263.net

Key words: circular RNA, micro RNA, microRNA-145, bladder cancer, microarray

\section{Introduction}

Bladder cancer ranks as the first and second most common urological malignancies in China and in the USA, respectively $(1,2)$. The characteristics of bladder cancer include high recurrence, morbidity and mortality rates $(1,2)$. With advances in surgical skills, three-year overall survival rates after radical cystectomy were less than $80 \%$ in China in 2015 (1). It is therefore crucial to determine the underlying mechanism of bladder carcinogenesis and identify novel molecular targets. To the best of our knowledge, conventional research on bladder cancer mainly focused on mRNAs; however, more attention has been paid to numerous non-coding RNAs, including microRNAs (miRNAs) and long noncoding RNAs (3-5). In particular, circular RNAs (circRNAs), which represent a novel type of noncoding RNA with notable regulatory potency (6-9), have attracted much interest. circRNAs can serve as miRNA sponges and influence the expression levels of miRNA-targeted transcripts (10). Furthermore, it was demonstrated that the circRNA/miRNA axes serve crucial roles in numerous tumorassociated signaling pathways, such as tight junction signaling, endocytosis and mTOR signaling pathways $(11,12)$. Recent studies have determined the function of certain circRNAs in bladder cancer (13-17). Li et al (15) found that Cdrlas was downregulated and sponged multiple miRNAs in bladder cancer, and demonstrated that it could exert anti-oncogenic functions by sponging microRNA-135a. In addition, Zhong et al (16) reported that circRNA-MYLK might function as competing endogenous RNA (ceRNA) for miR-29a, which could contribute to epithelial-mesenchymal transition and the development of bladder cancer by activating VEGFA/VEGFR2 and downstream Ras/ ERK signaling pathway. However, the role of all circRNAs in bladder cancer, and the number of circRNAs acting as miRNA sponges in bladder carcinoma remain unknown.

The present study aimed to investigate the expression pattern of circRNAs in four bladder cancer tissues and matched normal tissues by microarray analysis. The differential expression levels of representative circRNAs were further determined in 16 pairs of tissues using reverse transcription-quantitative polymerase chain reaction (RT-qPCR). The signaling pathways associated with the dysregulated circRNAs in bladder cancer tissues were therefore determined using Kyoto Encyclopedia of Genes and Genomes (KEGG) pathway analysis. A circRNA/ miRNA network was then constructed through bioinformatics methods. The results from the present study indicated that 
certain differentially expressed circRNAs may be considered as important therapeutic targets in bladder cancer.

\section{Materials and methods}

Patients and specimens. Four bladder cancer and matched normal samples were collected from four patients at the First Affiliated Hospital of Zhengzhou University between January 2016 and November 2016. The mean age was 62 year-old (range, 50-65 years). These specimens were used for the circRNA microarray. Additional 16 pairs of tissues from the Department of Urology of The First Affiliated Hospital of Zhengzhou University were used to validate circRNA expression by RT-qPCR. All the samples were collected from patients with advanced bladder cancer who received radical cystectomy without preoperative chemotherapy or radiotherapy. Tissue specimens were immediately stored in liquid nitrogen following surgical resection until further experiment.

RNA extraction. Total RNA was extracted from frozen specimens using TRIzol ${ }^{\circledR}$ (Invitrogen; Thermo Fisher Scientific, Inc.) according to the manufacturer's instructions. RNA concentration was determined by measuring the OD260 value with a NanoDrop spectrophotometer (NanoDrop Technologies; Thermo Fisher Scientific, Inc.) and the RNA integrity was assessed using electrophoresis.

Microarray hybridization. Microarray hybridization was performed as previously described (18). Briefly, total RNAs were digested with RNase R (Epicentre; Illumina, Inc.) to remove linear RNAs and enrich circular RNAs. Next, the enriched circular RNAs were amplified and transcribed into fluorescent cRNA utilizing a random priming method (Arraystar Super RNA Labeling kit; Arraystar). The labeled cRNAs were purified by RNeasy Mini kit (Qiagen GmbH). The concentration and specific activity of the labeled cRNAs (pmol Cy3/ $\mu \mathrm{g}$ cRNA) were measured by NanoDrop ND-1000. Each labeled cRNA $(1 \mu \mathrm{g})$ was fragmented by adding $5 \mu \mathrm{l} 10 \mathrm{X}$ blocking agent and $1 \mu \mathrm{l} 25 \mathrm{X}$ fragmentation buffer, then heated the mixture at $60^{\circ} \mathrm{C}$ for $30 \mathrm{~min}$. Finally $25 \mu \mathrm{l} 2 \mathrm{X}$ hybridization buffer was added to dilute the labeled cRNA. Hybridization solution $(50 \mu \mathrm{l})$ was dispensed into the gasket slide and assembled to the circRNA expression microarray slide. The slides were incubated for $17 \mathrm{~h}$ at $65^{\circ} \mathrm{C}$ in an Agilent Hybridization oven. The hybridized arrays were washed, fixed and scanned using the Agilent Scanner (Agilent Technologies Inc., part number G2505C). The acquired array images were analyzed by Agilent Feature Extraction software (version 10.5.1.1; Agilent Technologies Inc.,). Microarray hybridization and data collection were carried out by Kangchen BioTech Co., Ltd.

Microarray data analysis. Microarray data analysis was performed as previously described (19). Briefly, circRNAs expression profiles between bladder cancer and normal control samples were compared by calculating the fold change for each circRNA (19). CircRNAs with fold changes $\geq 1.5$ and $P \leq 0.05$ were considered as significantly differentially expressed.

$R T-q P C R$. Total RNA was extracted from frozen tissues in liquid nitrogen using TRIzol (Invitrogen; Thermo Fisher
Scientific, Inc.). The expression levels of the circRNAs were assessed by RT-qPCR as previously described (19). According to manufacturer's instructions, M-MLV reverse transcriptase (Invitrogen; Thermo Fisher Scientific, Inc.) was used for synthesizing cDNA following RNA extraction. The expression level of the circRNAs was assessed by RT-qPCR using SYBR Green assay (Arraystar Inc.). Specific divergent primers (Table SI) were designed to amplify the circular transcripts. PCR was carried out in a $10-\mu 1$ reaction volume, including $2 \mu \mathrm{l}$ of cDNA, $5 \mu \mathrm{l} 2 \mathrm{X}$ Master mix (Arraystar Inc.), $0.5 \mu \mathrm{l}$ of forward primer $(10 \mu \mathrm{M}), 0.5 \mu 1$ of reverse primer $(10 \mu \mathrm{M})$ and $2 \mu 1$ of double distilled water. The reaction was set at $95^{\circ} \mathrm{C}$ for $10 \mathrm{~min}$ for pre-denaturation, followed by $95^{\circ} \mathrm{C}$ for $10 \mathrm{sec}$ and at $60^{\circ} \mathrm{C}$ for $60 \mathrm{sec}$ repeating 40 cycles. $\beta$-actin was used as a reference. Both target and reference were amplified in triplicate wells. The relative expression levels of the circRNAs were normalized to the endogenous control $\beta$-actin and expressed as $2^{-\Delta \Delta \mathrm{Cq}}$.

Delineation of circRNA/miRNA interactions. circRNA/ miRNA interactions were predicted using miRanda (http:// www.microrna.org/microrna/home.do) and TargetScan tools (http://www.targetscan.org). The microRNA response elements (MREs) on the circRNAs were therefore determined and the corresponding miRNAs were identified. Cytoscape 3.0 (https://cytoscape.org/) was used to generate the circRNA/ miRNA network diagram.

Statistical analysis. Student's t-tests (two-tailed) was used to compare the expression levels of circRNAs between bladder cancer and normal control samples. $\mathrm{P}<0.05$ was considered to indicate a statistically significant difference.

\section{Results}

Identification of differentially expressed circRNAs in bladder cancer samples. The circRNA microarray was used to investigate the circRNA profiles in bladder cancer and matched normal samples. A box plot was drawn for visualizing the distribution the intensities of all the datasets following normalization, and observed that the distribution of $\log _{2}$ ratios was similar in all the tested samples (Fig. 1A). Hierarchical clustering was used for generating an overview of circRNA expression profiles between the two groups. The circRNA expression patterns were distinguishable between experiment and control groups (Fig. 1B). Scatter plots were performed to evaluate differential circRNA expression between two different conditions. Differentially changed circRNAs were identified through fold change filtering (Fig. 1C). Volcano plots were used to visualize the difference in the expression of circRNAs in bladder cancer tissues and paired adjacent normal tissues. The volcano plot filtering displayed differentially expressed circRNAs between the two groups (Fig. 1D).

Following circRNA microarray analysis, significant differential expression was observed in 299 circRNAs, of which 89 circRNAs were downregulated and 210 circRNAs were upregulated (Table SII). The results from microarray demonstrated the distribution of dysregulated circRNAs on human chromosomes. The upregulated and downregulated circRNAs were further summarized and the differentially expressed circRNAs on human chromosomes were delineated (Fig. 2A). 


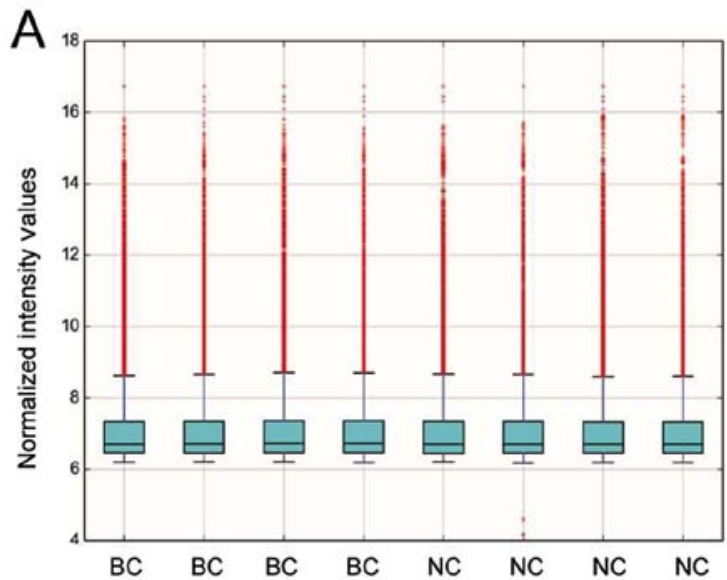

B

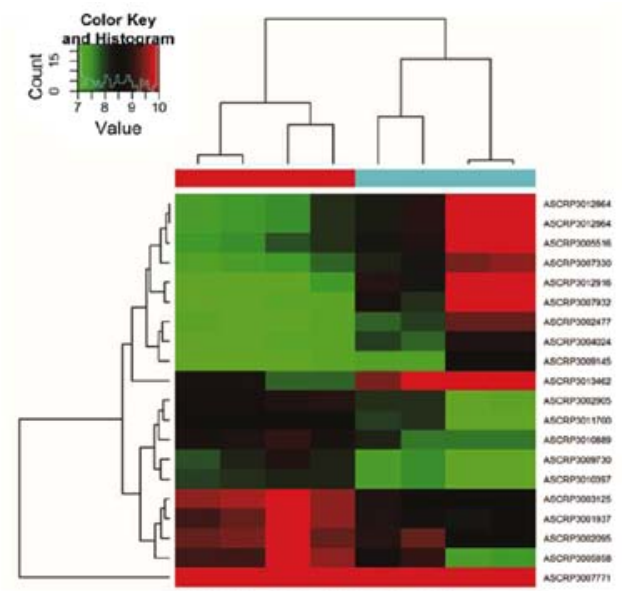

NC NC NC NC BC BC BC BC
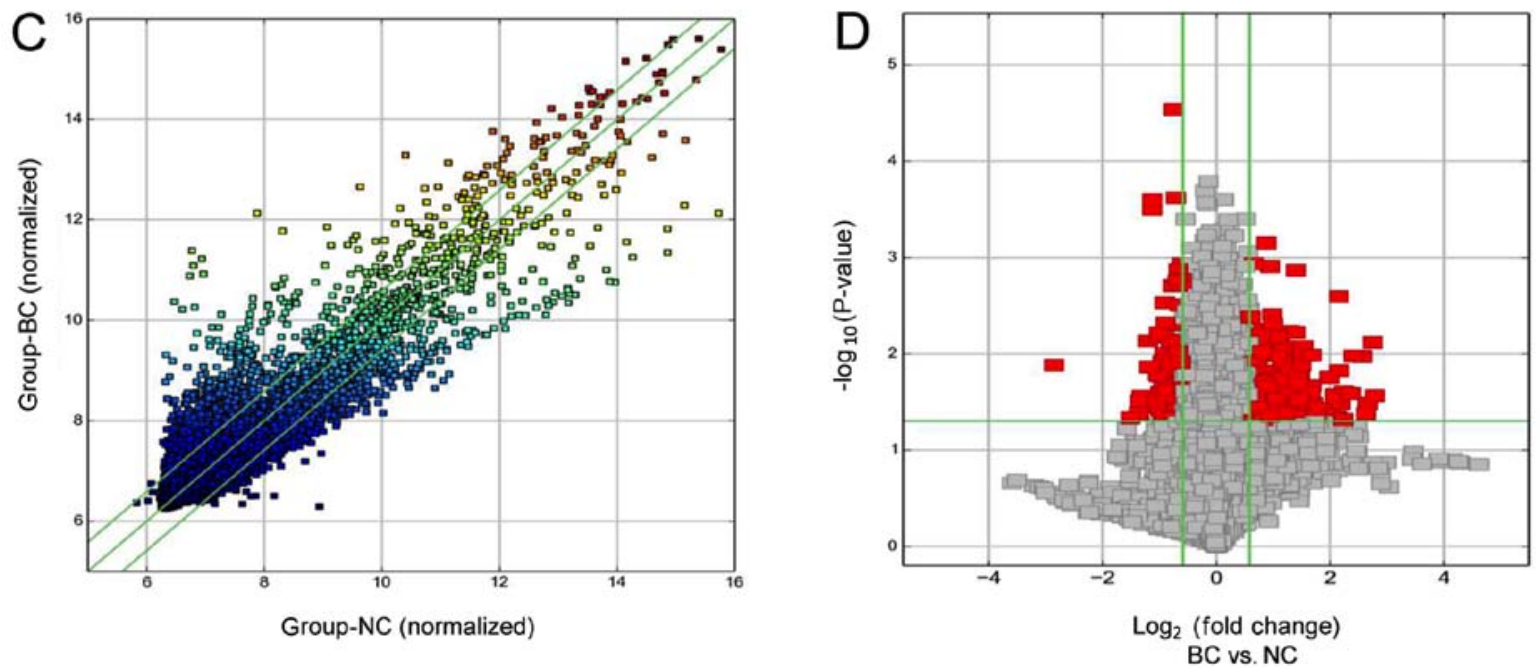

Figure 1. Bioinformatics analysis of dysregulated circRNAs and mRNAs in BC and NC samples. (A) Box plot of 8 tissue samples and their normalized intensity values. The distribution of circRNAs was almost similar in all samples. (B) Unsupervised hierarchical cluster analysis of unique circRNA expression profile of the 8 samples in red (low relative expression) or green (high relative expression) scale. (C) Scatter plot of adjacent non-tumor tissues (normalized) vs. bladder cancer tissues (normalized). The green lines are fold change lines. The circRNAs above the top green line and below the bottom green line indicate more than 1.5 fold change of circRNAs between the two samples compared. (D) Volcano plot of $\log _{2}$ (fold change) and $-\log _{10}($ P-value). The vertical lines correspond to 1.5 fold up and down, respectively, and the horizontal line represents a P-value of 0.05 . The red point in the plot represents the differentially expressed circRNAs with statistical significance. BC, bladder cancer; circRNA, circular RNA; NC, normal control.
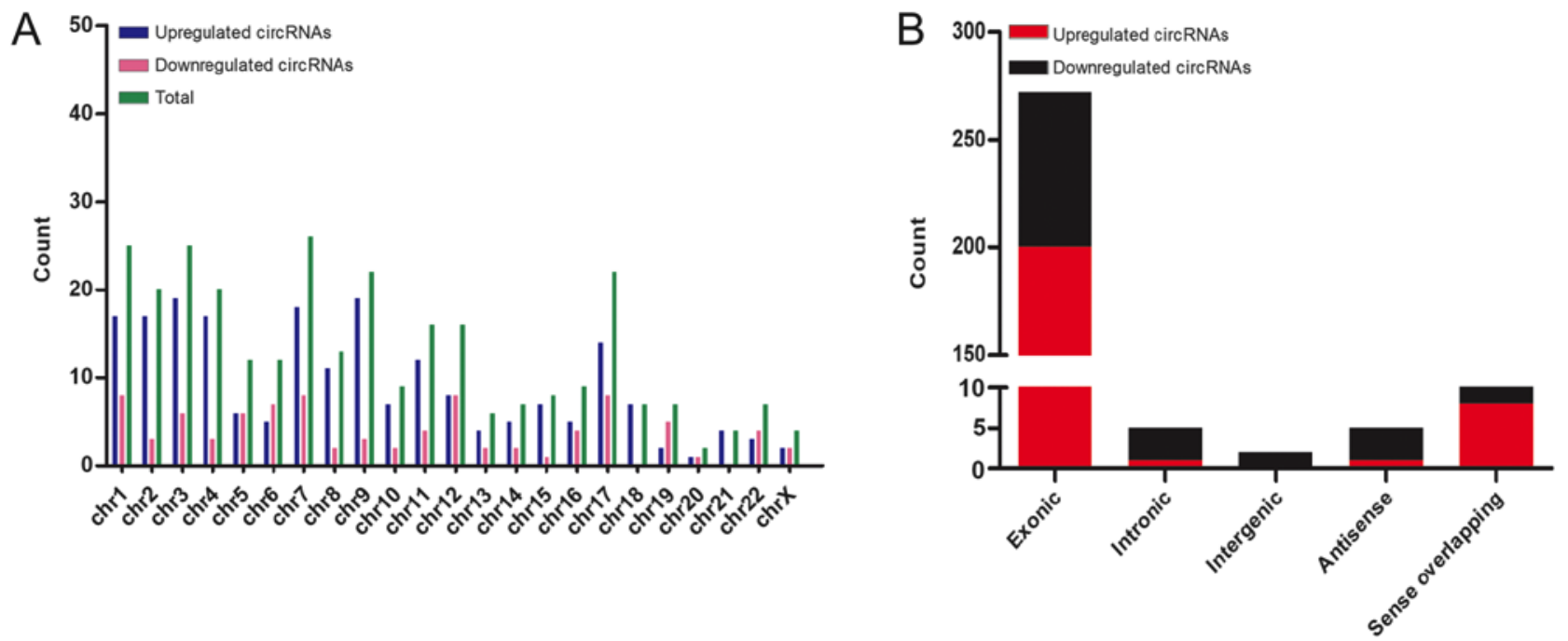

Figure 2. Characterization of circRNA expression profiles in bladder cancer and matched normal samples. (A) Distribution of dysregulated circRNAs on human chromosomes. (B) circRNA category based on origin. Most of the dysregulated circRNAs were transcribed by exons. circRNA, circular RNA. 
A
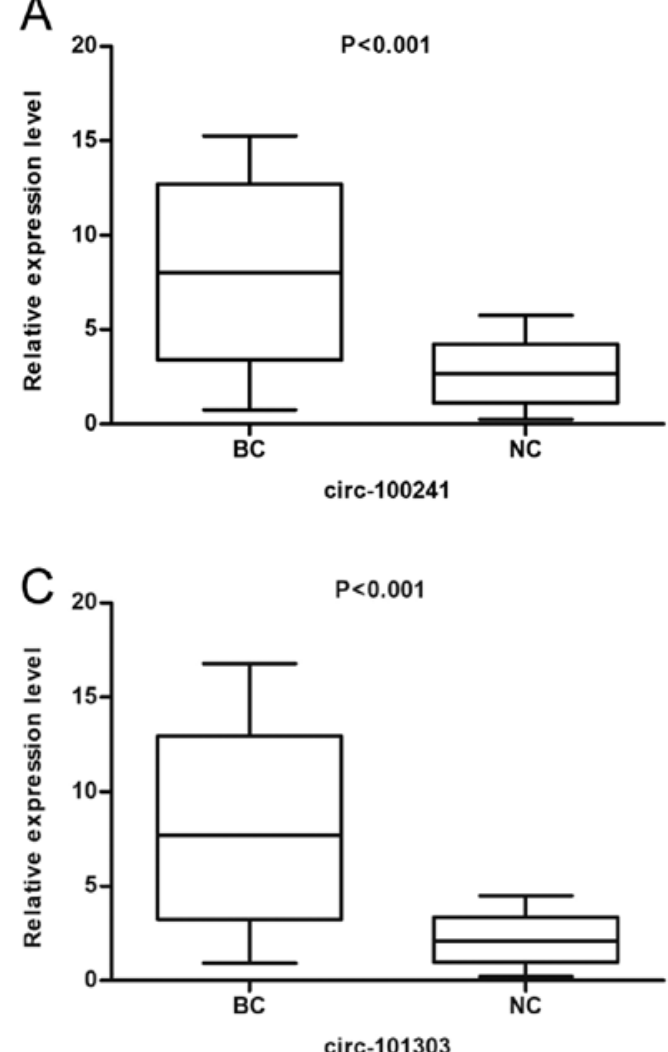

B
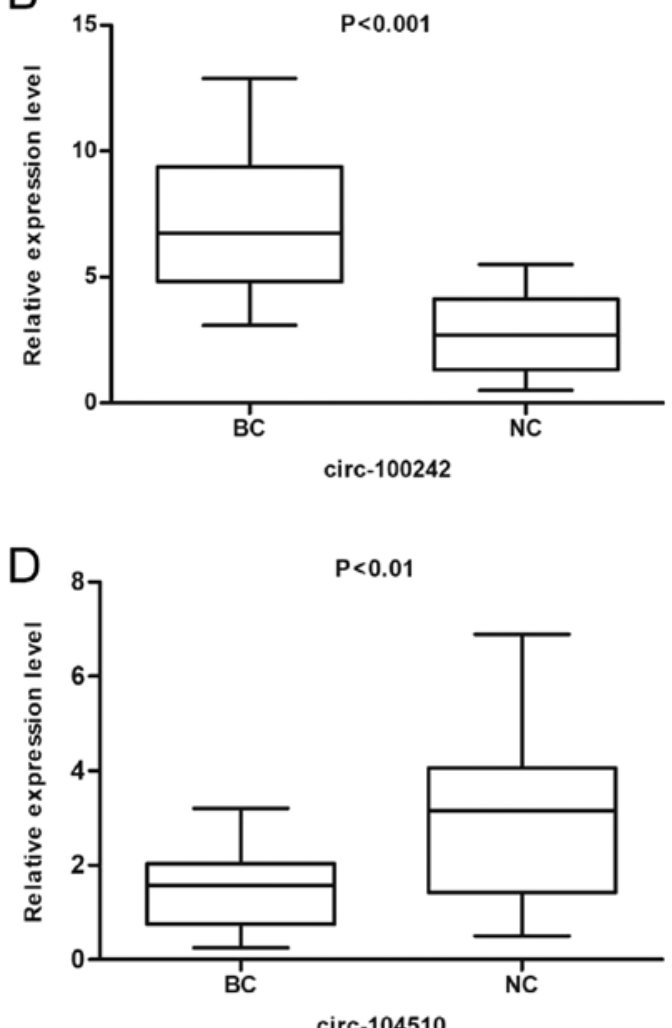

Figure 3. Expression of (A) circ-100241, (B) circ-100242, (C) circ-101303 and (D) circ-104510 assessed by reverse transcription-quantitative polymerase chain reaction. BC, bladder cancer; circRNA, circular RNA; NC, normal control.

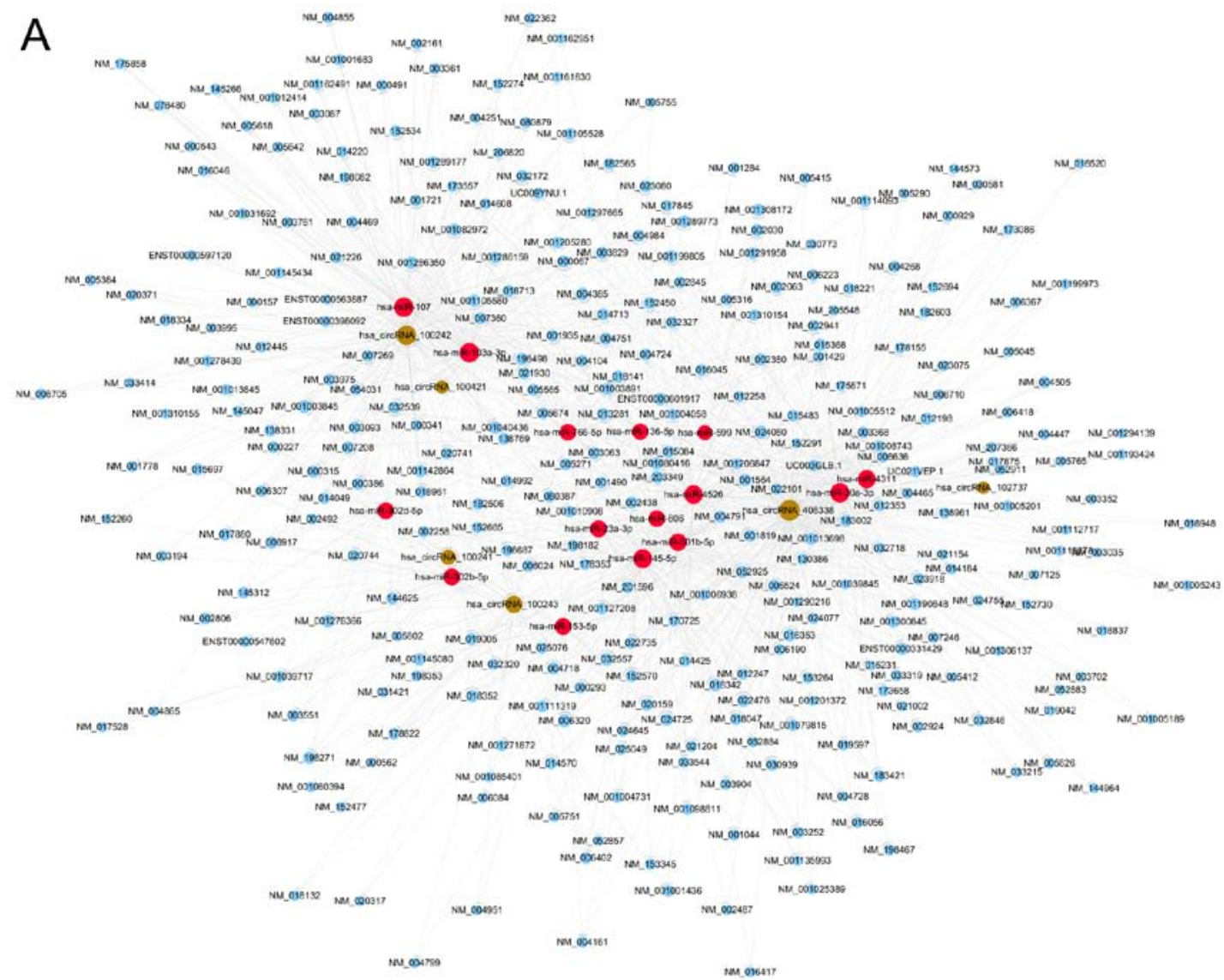

Figure 4. circRNA/miRNA network analysis. (A) The circRNA/miRNA network in bladder carcinoma included circRNAs (yellow nodes), their target miRNAs (red nodes) and the associated mRNAs (blue nodes). 


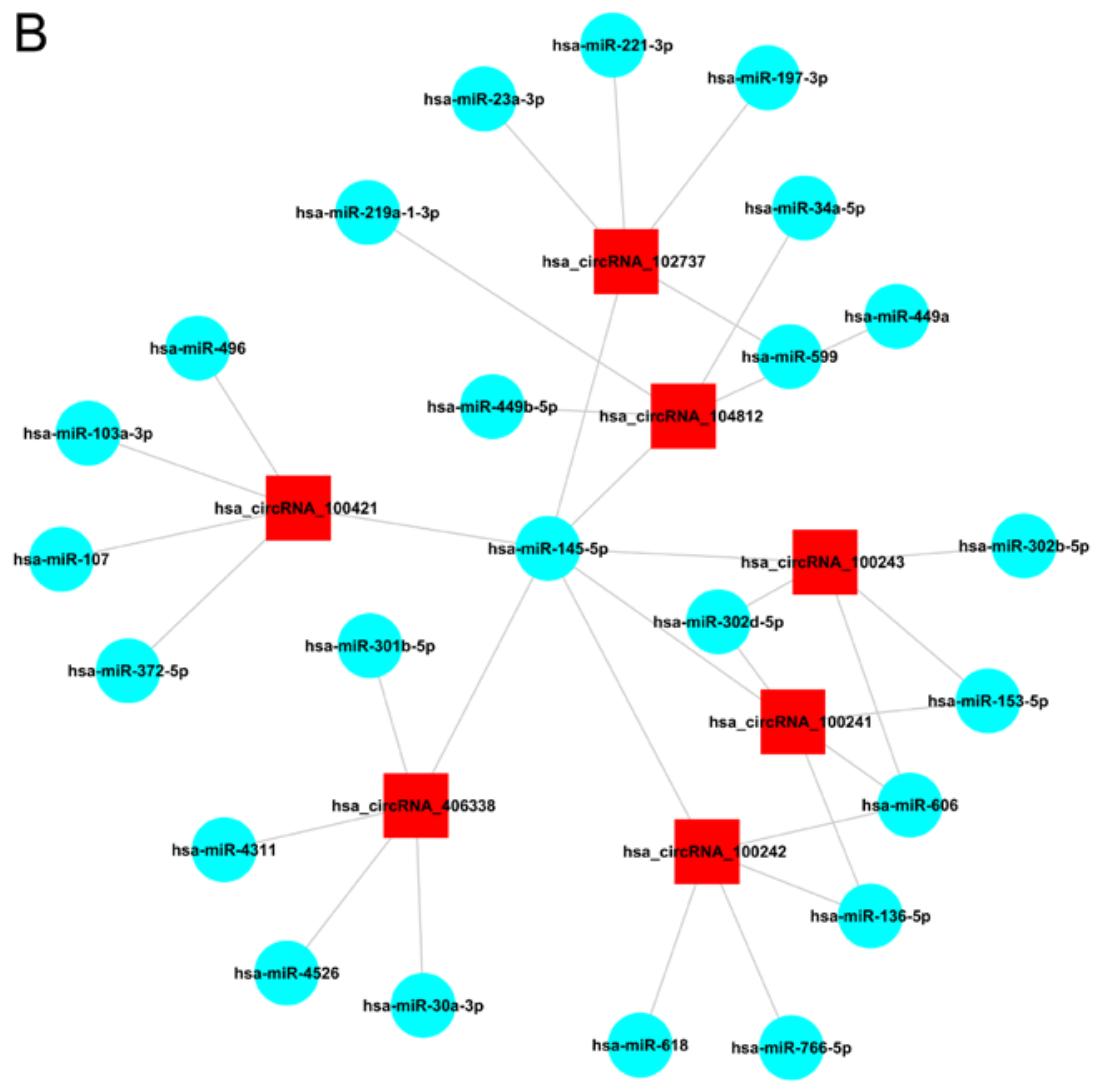

Figure 4. Continued. (B) Magnified circRNA/miRNA network of several circRNAs (in red) and their target miRNAs (in blue) in bladder cancer sample. circRNA, circular RNA; miRNA, microRNA.

Most of the dysregulated circRNAs were transcribed by exons (Fig. 2B).

Validation of the differential expression levels of circRNAs. A total of four dysregulated circRNAs were randomly selected to confirm the microarray findings by using RT-qPCR. The results demonstrated that hsa_circ_100241, hsa_circ_100242 and hsa_circ_101303 were validated as significantly upregulated in bladder cancer samples, whereas hsa_circ_104510 was significantly downregulated in bladder cancer samples compared with adjacent normal tissues (Fig. 3). These results were consistent with findings from the microarray analysis.

Construction of the circRNA/miRNA interaction network. The circRNA/miRNA interaction was theoretically predicted through Arraystar's home-made miRNA target prediction software based on TargetScan and miRanda. Cytoscape was used to depict an entire network of circRNA/miRNA interaction (Fig. 4A). The part of the graph was then enlarged to clearly display several circRNAs and their target miRNAs in bladder cancer (Fig. 4B). One confirmed circRNA (hsa_circ_100242) was annotated in detail with circRNA/miRNA interaction information, indicating that hsa_circ_100242 had microRNA response element of miR-145-5p (Fig. 5).

Bioinformatics analyses. The results from KEGG pathway analysis demonstrated that 10 signaling pathways were associated with the dysregulated circRNAs, including 'MAPK pathway', 'transcriptional misregulation in cancer', 'aldosterone synthesis and secretion', 'TGF- $\beta$ pathway', 'Hippo pathway', 'proteoglycans in cancer', 'axon guidance', 'FoxO signaling in cancer', 'endocytosis' and 'Rapl pathway'. In particular, 'MAPK signaling pathway' was the most important pathway of the dysregulated circRNAs in bladder cancer samples (Fig. 6).

\section{Discussion}

The present study determined the expression profiles of circRNAs in advanced bladder cancer samples by using circRNA microarray. The analysis revealed 210 upregulated circRNAs and 89 downregulated circRNAs in bladder cancer tissues. Furthermore, the 'MAPK signaling pathway' was the most significant pathway for the differentially expressed circRNAs in bladder cancer. According to the results from circRNA microarray and our previous study (20), hsa_circ_100242 may be involved in bladder cancer initiation and progression by sponging miR-145.

Previous studies have reported that circRNAs could be considered as ceRNA molecules or miRNA sponges and serve a crucial role in certain diseases (such as pathological hypertrophy and glioma) by influencing gene expression (7,10,21-24). For instance, Wang et al (21) demonstrated that heart-related circRNAs (HRCRs) could affect miR-223 activity in cardiac hypertrophy by acting as an endogenous miR-223 sponge. HRCRs may therefore represent novel therapeutic targets for treating cardiac hypertrophy. In the present study, circRNA/ miRNA interactions were also predicted on the basis of 

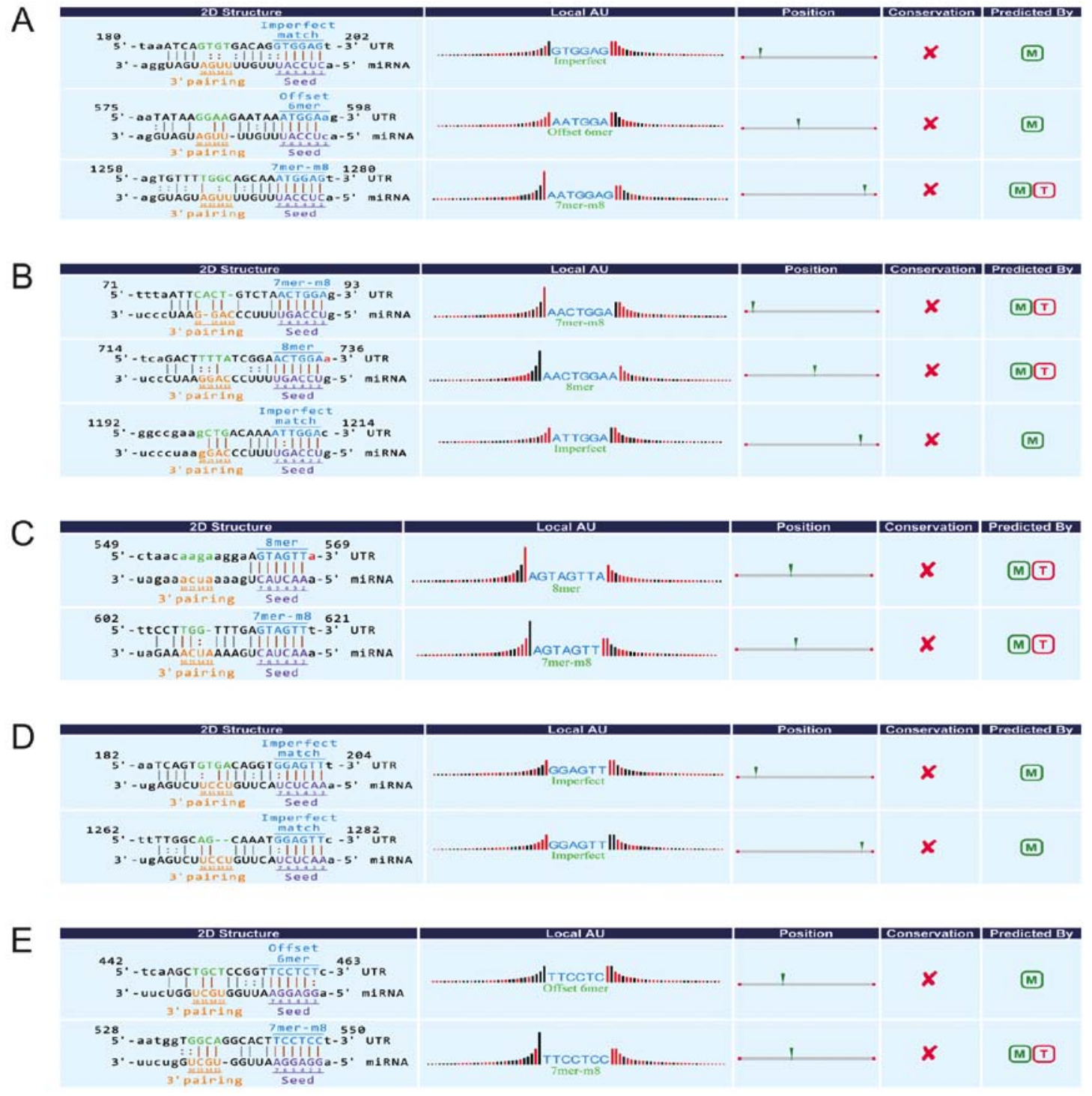

Figure 5. Snapshot of the detailed annotation of circRNA/miRNA interactions. (A) circ_100242 vs. miR-136-5p. (B) circ_100242 vs. miR-145-5p. (C) circ_100242 vs. miR-606. (D) circ_100242 vs. miR-618. (E) circ_100242 vs. miR-766-5p. circRNA, circular RNA; M, circRNA/miRNA interaction predicted by miRanda; $\mathrm{miR} / \mathrm{miRNA}$, microRNA; T, circRNA/miRNA interaction predicted by TargetScan.

conserved seed sequence matches using miRanda and TargetScan tools. In addition, the circRNA/miRNA interaction network that was constructed in this study may provide novel evidence for further research on ceRNAs involving dysregulated circRNAs.

The present study demonstrated that hsa_circ_100242 was significantly upregulated in bladder cancer samples compared with matched normal samples. Our previous study demonstrated that miR-145 could directly target the 3'-untranslated region of the type 1 insulin-like growth factor receptor (IGF-IR) in human bladder cancer sample (20). In addition, results from small interfering RNA- and miR-145-mediated IGF-IR knockdown experiments revealed that miR-145 could promote bladder cancer cell apoptosis and inhibit its cell proliferation and migration via suppression of IGF-IR expression (20). Subsequently, hsa_circ_100242 may be implicated in bladder cancer initiation and progression by targeting miR-145-5p. The role of hsa_circ_100242 in bladder cancer requires therefore further investigation.
The present study presented some limitations. Firstly, bladder cancer and matched normal tissues were all collected from one hospital. Secondly, the small sample size of bladder cancer tissues used for the microarray analysis was not large enough to draw any definitive conclusions. Thirdly, only the expression profiles of circRNAs in bladder cancer and matched normal samples were compared. Of equal importance is the differential circRNA expression profiles in bladder cancer patients of different genders, stages or grades. In future investigation, a higher number of tissues from various hospitals should therefore be collected to carry out a deeper analysis. Fourthly, circRNAs expression in blood and urine might be also crucial for the identification of potent biomarkers for earlier diagnosis of bladder cancer. Blood and urine samples from patients with bladder cancer are therefore currently being collected for future investigation. Experiments in bladder cancer cell lines will also be performed. Furthermore, following RNA interference and transfection of adenovirus vector plasmid in bladder cancer cell lines, the role of circRNA in bladder cancer and the determination of 


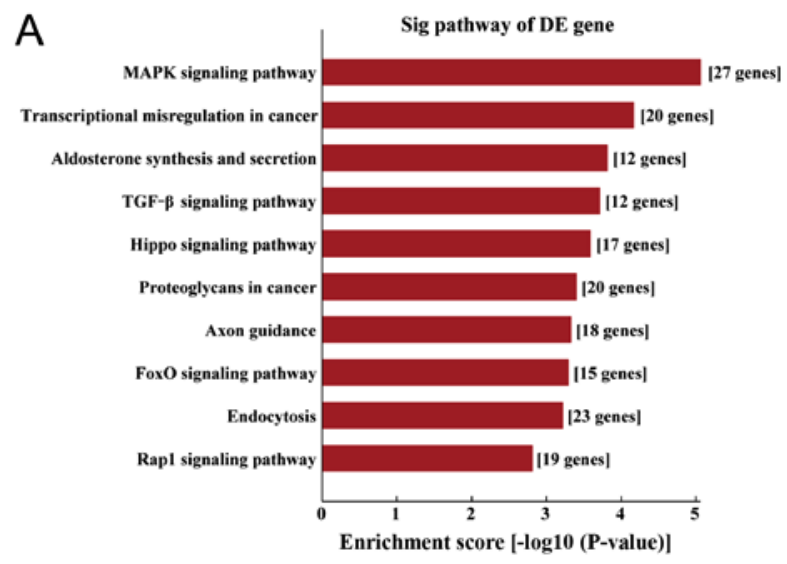

B

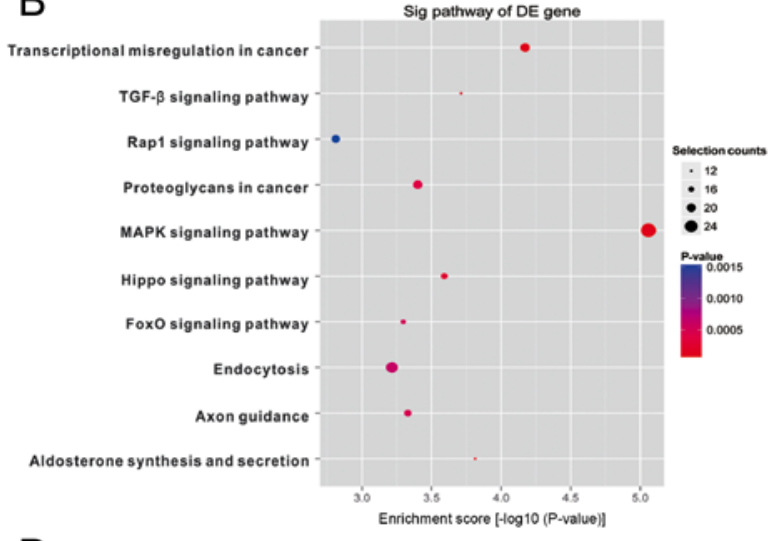

C

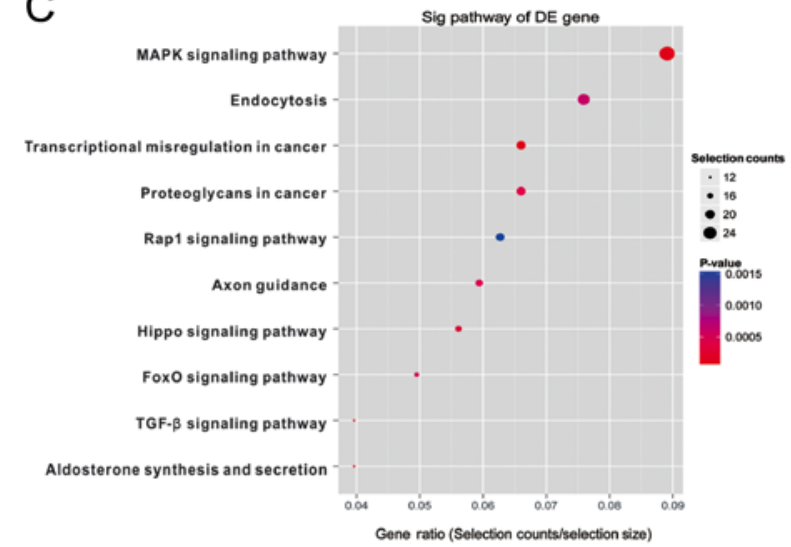

D

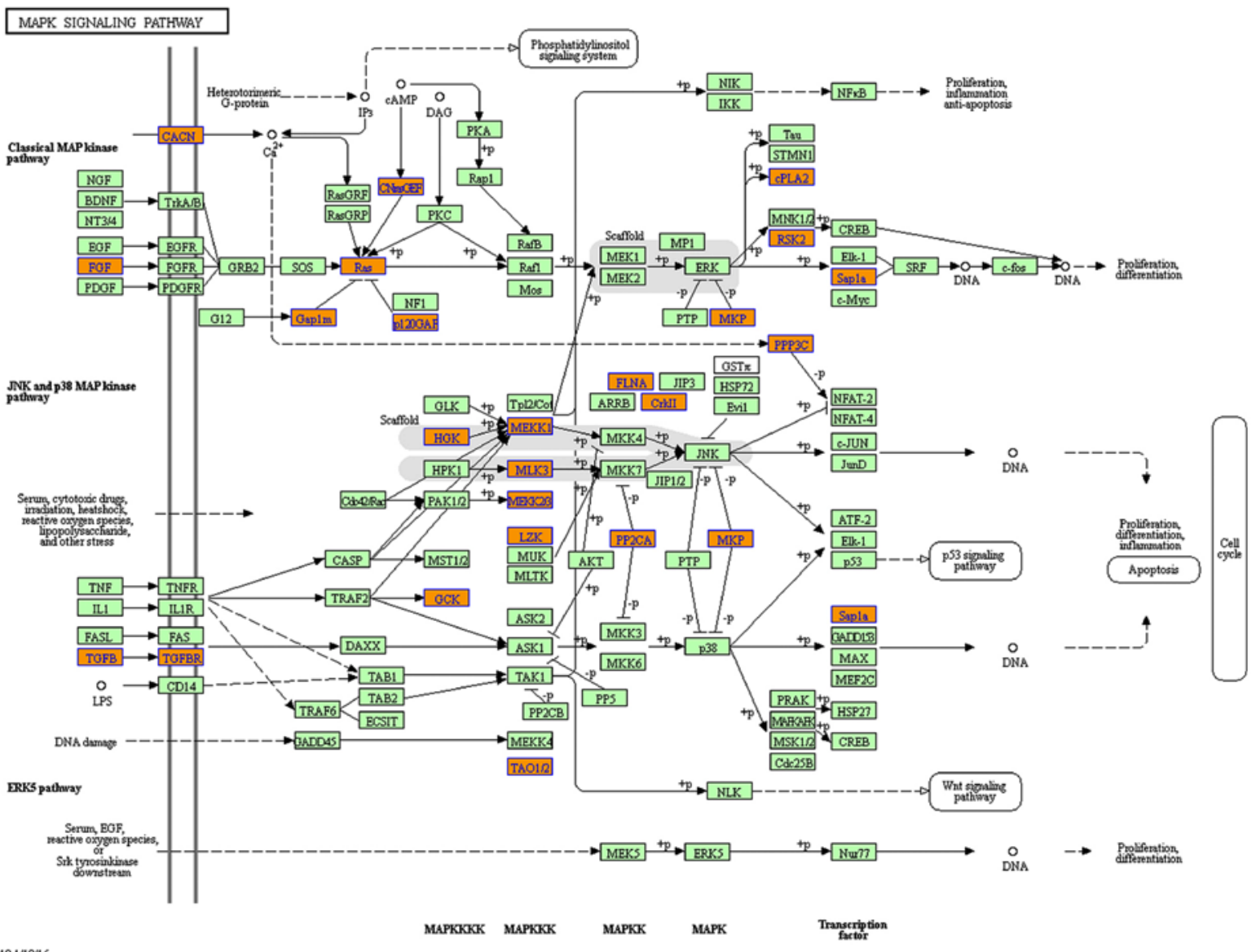

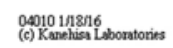

Figure 6. Kyoto Encyclopedia of Genes and Genomes pathway analysis of bladder cancer samples. (A) Pathway barplot explanation (EnrichmentScore): The bar plot shows the top ten enrichment score [- $\log _{10}(\mathrm{P}$-value)] value of the significant enrichment pathway. (B) Pathway dotplot explanation (EnrichmentScoreDotPlot): The dot plot shows the top ten enrichment score [- $\log _{10}$ (Pvalue)] value of the significant enrichment pathway. (C) Pathway dotplot explanation (GeneRatioDotPlot): The dot plot shows the gene ratio value of the top ten most significant enrichment pathways. (D) MAPK signaling pathway. DE, differentially expressed. 
the regulatory mechanism of circRNA on biological activity of bladder cancer cells will be further investigated.

The present study demonstrated that circRNAs were differentially expressed in bladder cancer tissues compared with matched normal samples. Subsequently, pathway analysis was performed, and binding miRNAs were predicted for the dysregulated circRNAs of bladder cancer tissues. The results revealed that 'MAPK signaling pathway' was the most important pathway of the differentially expressed circRNAs in bladder cancer samples. In addition, hsa_circ_100242 may be involved in bladder cancer initiation and progression by sponging miR-145. These findings may lay the foundation for future studies on circRNAs in bladder cancer.

\section{Acknowledgements}

Not applicable.

\section{Funding}

The present study was funded by the National Natural Science Foundation of China (grant no. 81702503).

\section{Availability of data and materials}

Not applicable.

\section{Authors' contributions}

ZZ and XZ: Conception and design of the study, and final approval of the manuscript. FC, JL and JW: Acquisition, analysis and interpretation of data.

\section{Ethics approval and consent to participate}

The study was granted an exemption from requiring ethic approval from the Ethics Committee of our institution. Patients provided informed consent prior to the study.

\section{Patient consent for publication}

Not applicable.

\section{Competing interests}

The authors declare that they have no competing interests.

\section{References}

1. Chen W, Zheng R, Baade PD, Zhang S, Zeng H, Bray F, Jemal A, Yu XQ and He J: Cancer statistics in China, 2015. CA Cancer J Clin 66: 115-132, 2016.

2. Siegel RL, Miller KD and Jemal A: Cancer statistics, 2018. CA Cancer J Clin 68: 7-30, 2018.

3. Pasquinelli AE: MicroRNAs and their targets: Recognition, regulation and an emerging reciprocal relationship. Nat Rev Genet 13: 271-282, 2012.
4. Rupaimoole R and Slack FJ: MicroRNA therapeutics: Towards a new era for the management of cancer and other diseases. Nat Rev Drug Discov 16: 203-222, 2017.

5. Li Y and Wang X: Role of long noncoding RNAs in malignant disease (Review). Mol Med Rep 13: 1463-1469, 2016.

6. Memczak S, Jens M, Elefsinioti A, Torti F, Krueger J, Rybak A, Maier L, Mackowiak SD, Gregersen LH, Munschauer M, et al: Circular RNAs are a large class of animal RNAs with regulatory potency. Nature 495: 333-338, 2013.

7. Qu S, Yang X, Li X, Wang J, Gao Y, Shang R, Sun W, Dou K and Li H: Circular RNA: A new star of noncoding RNAs. Cancer Lett 365: 141-148, 2015.

8. Li Y, Zheng Q, Bao C, Li S, Guo W, Zhao J, Chen D, Gu J, He X, and Huang S: Circular RNA is enriched and stable in exosomes: A promising biomarker for cancer diagnosis. Cell Res 25: 981-984, 2015

9. Guo S, Xu X, Ouyang Y, Wang Y, Yang J, Yin L, Ge J and Wang H: Microarray expression profile analysis of circular RNAs in pancreatic cancer. Mol Med Rep 17: 7661-7671, 2018.

10. Hansen TB, Jensen TI, Clausen BH, Bramsen JB, Finsen B, Damgaard CK and Kjems J: Natural RNA circles function as efficient microRNA sponges. Nature 495: 384-388, 2013.

11. Liu BH, Zhang BB, Liu XQ, Zheng S, Dong KR and Dong R: Expression profiling identifies circular RNA signature in hepatoblastoma. Cell Physiol Biochem 45: 706-719, 2018.

12. Qian Y, Lu Y, Rui C, Qian Y, Cai M and Jia R: Potential significance of circular RNA in human placental tissue for patients with preeclampsia. Cell Physiol Biochem 39: 1380-1390, 2016.

13. Yang X, Yuan W, Tao J, Li P, Yang C, Deng X, Zhang X, Tang J, Han J, Wang J, et al: Identification of circular RNA signature in bladder cancer. J Cancer 8: 3456-3463, 2017.

14. Li M, Liu Y, Zhang X, Liu J and Wang P: Transcriptomic analysis of high-throughput sequencing about circRNA, lncRNA and mRNA in bladder cancer. Gene 677: 189-197, 2018.

15. Li P, Yang X, Yuan W, Yang C, Zhang X, Han J, Wang J, Deng X, Yang H, Li P, et al: CircRNA-cdrlas exerts anti-oncogenic functions in bladder cancer by sponging MicroRNA-135a. Cell Physiol Biochem 46: 1606-1616, 2018.

16. Zhong Z, Huang M, Lv M, He Y, Duan C, Zhang L and Chen J: Circular RNA MYLK as a competing endogenous RNA promotes bladder cancer progression through modulating VEGFA/ VEGFR2 signaling pathway. Cancer Lett 403: 305-317, 2017.

17. Xie F, Li Y, Wang M, Huang C, Tao D, Zheng F, Zhang H, Zeng F, Xiao X and Jiang G: Circular RNA BCRC-3 suppresses bladder cancer proliferation through miR-182-5p/p27 axis. Mol Cancer 17: 144, 2018.

18. Gou Q, Wu K, Zhou JK, Xie Y, Liu L and Peng Y: Profiling and bioinformatic analysis of circular RNA expression regulated by c-Myc. Oncotarget 8: 71587-71596, 2017.

19. Zhong Z, Lv M and Chen J: Screening differential circular RNA expression profiles reveals the regulatory role of circTCF25miR-103a-3p/miR-107-CDK6 pathway in bladder carcinoma. Sci Rep 6: 30919, 2016.

20. Zhu Z, Xu T, Wang L, Wang X, Zhong S, Xu C and Shen Z: MicroRNA-145 directly targets the insulin-like growth factor receptor I in human bladder cancer cells. FEBS Lett 588: 3180 3185,2014

21. Wang K, Long B, Liu F, Wang JX, Liu CY, Zhao B, Zhou LY, Sun T, Wang M, Yu T, et al: A circular RNA protects the heart from pathological hypertrophy and heart failure by targeting miR-223. Eur Heart J 37: 2602-2611, 2016.

22. Zheng Q, Bao C, Guo W, Li S, Chen J, Chen B, Luo Y, Lyu D, Li Y, Shi G, et al: Circular RNA profiling reveals an abundant circHIPK 3 that regulates cell growth by sponging multiple miRNAs. Nat Commun 7: 11215, 2016.

23. Jin P, Huang Y, Zhu P, Zou Y, Shao T and Wang O: CircRNA circHIPK3 serves as a prognostic marker to promote glioma progression by regulating miR-654/IGF2BP3 signaling. Biochem Biophys Res Commun 503: 1570-1574, 2018.

24. Li Y, Wan B, Liu L, Zhou L and Zeng Q: Circular RNA circMTO1 suppresses bladder cancer metastasis by sponging miR-221 and inhibiting epithelial-to-mesenchymal transition. Biochem Biophys Res Commun 508: 991-996, 2019. 